\title{
P-glycoprotein mediated efflux limits substrate and drug uptake in a preclinical brain metastases of breast cancer model
}

\author{
Chris E. Adkins' ${ }^{1}$ Rajendar K. Mittapalli ${ }^{1}$, Vamshi K. Manda' ${ }^{1}$, Mohamed I. Nounou ${ }^{1}$, Afroz S. Mohammad' , \\ Tori B. Terrell' , Kaci A. Bohn' ${ }^{1}$, Celik Yasemin ${ }^{2}$, Tiffany R. Grothe ${ }^{2}$, Julie A. Lockman ${ }^{2}$ and Paul R. Lockman ${ }^{1}$ * \\ ' Department of Pharmaceutical Sciences, School of Pharmacy, Texas Tech University Health Sciences Center, Amarillo, TX, USA \\ ${ }^{2}$ Department of Life, Earth and Environmental Sciences, West Texas A\&M University, Canyon, TX, USA
}

\section{Edited by:}

Chiara Riganti, University of Torino,

Italy

\section{Reviewed by:}

Laura Annovazzi, Policlinico di Monza

Foundation, Italy

Valentina Caldera,

Neuro-Bio-Oncology Center,

Policlinico di Monza Foundation, Italy

\section{*Correspondence:}

Paul R. Lockman, Department of Pharmaceutical Sciences, School of Pharmacy, Texas Tech University Health Sciences Center, Amarillo Research Building, 1406 South Coulter, Amarillo, TX 79106-1712, USA e-mail:paul.lockman@ttuhsc.edu
The blood-brain barrier (BBB) is a specialized vascular interface that restricts the entry of many compounds into brain. This is accomplished through the sealing of vascular endothelial cells together with tight junction proteins to prevent paracellular diffusion. In addition, the BBB has a high degree of expression of numerous efflux transporters which actively extrude compounds back into blood. However, when a metastatic lesion develops in brain the vasculature is typically compromised with increases in passive permeability (blood-tumor barrier; BTB). What is not well documented is to what degree active efflux retains function at the BTB despite the changes observed in passive permeability. In addition, there have been previous reports documenting both increased and decreased expression of P-glycoprotein (P-gp) in lesion vasculature. Herein, we simultaneously administer a passive diffusion marker $\left({ }^{14} \mathrm{C}-\mathrm{AlB}\right)$ and a tracer subject to P-gp efflux (rhodamine 123) into a murine preclinical model of brain metastases of breast cancer. We observed that the metastatic lesions had similar expression $(p>0.05 ; n=756-1214$ vessels evaluated) at the BBB and the BTB. Moreover, tissue distribution of R123 was not significantly $(p>0.05)$ different between normal brain and the metastatic lesion. It is possible that the similar expression of P-gp on the BBB and the BTB contribute to this phenomenon. Additionally we observed P-gp expression at the metastatic cancer cells adjacent to the vasculature which may also contribute to reduced R123 uptake into the lesion. The data suggest that despite the disrupted integrity of the BTB, efflux mechanisms appear to be intact, and may be functionally comparable to the normal BBB. The BTB is a significant hurdle to delivering drugs to brain metastasis.

Keywords: drug resistance, chemotherapy, autoradiography, fluorescence microscopy, tumor

\section{INTRODUCTION}

The successful treatment of central nervous system (CNS) tumors and metastases using chemotherapy depends on the ability of therapeutic concentrations of drug to cross the blood-brain barrier (BBB). More than 98\% of potential CNS active anticancer drugs fail in preclinical work and or clinical trials because of inadequate BBB penetration (Pardridge, 2007). Clinically this results in many anticancer agents failing to substantially reduce tumor burden and or significantly prolong survival (Deeken and Loscher, 2007).

The microvasculature of the brain is a unique anatomical structure which serves as a homeostatic and regulatory barrier between the blood and the brain parenchyma (Hawkins and Davis, 2005). Specifically, endothelial cells that line the blood vessels of the brain capillaries are fused together by numerous tight junction protein complexes, which restrict blood components from passively diffusing between the cell margins to gain entry into brain. The tight junction protein complexes consist of a number of proteins such as zonula occludins, junctional adhesion molecules, and claudins which function as a unit to seal the endothelia margins. Further the outside of the brain capillary is surrounded by astrocytic foot processes and pericytes that also contribute to the restriction of paracellular diffusion (Abbott et al., 2010).

Further restricting the brain entry of a large number of drugs and drug classes are efflux transporters at the BBB. Efflux transporters are richly expressed in the brain vasculature and have been shown to restrict the accumulation of antiepliptics, antidepressants, and antipsychotics (Schinkel et al., 1995; Loscher and Potschka, 2005). Multiple efflux transporters at the BBB act to actively extrude or prevent drug accumulation into brain, these include P-glycoprotein (P-gp; ABCB1) (Schinkel et al., 1996), breast cancer resistant protein (BCRP; ABCG2; Polli et al., 2009), multidrug resistance associated proteins (MRP; ABCC16; Breedveld et al., 2005), and organic anion transporters (OATs; Hagenbuch and Meier, 2004).

The net effect of the anatomical and molecular features of the $\mathrm{BBB}$ is that to a large degree it restricts drug movement from blood into brain. But some drugs are able to penetrate the BBB. Drug and/or solute permeation across the BBB is mostly limited 
to low molecular weight lipid-soluble molecules. Molecules that are large (typically $>700 \mathrm{Da}$ ), protein bound or are hydrophilic will have difficulty crossing the BBB and accumulating in brain to a sufficient degree (Lipinski et al., 2001).

However, the vasculature within a brain tumor is different from the normal BBB. Previously it has been shown that the blood-tumor barrier (BTB) vasculature has disrupted integrity compared to the intact BBB. This disruption can allow small molecule to accumulate into lesions up to 30-100-fold more than the accumulation of the molecule in normal brain (Lockman et al., 2010; Taskar etal., 2012). While the degree of breakdown at the BTB does correlate with increases in drug uptake it is not clearly defined whether efflux pumps continue to limit drug uptake into metastatic lesions (Gallo et al., 2003). It has been previously shown that the BTB expresses P-gp (Cordon-Cardo et al., 1990); however, the expression of P-gp may be variable among different tumors types (Henson et al., 1992). In addition to P-gp expression at the BTB, many cancers have been shown to express functional P-gp in vivo which may restrict the cellular accumulation of chemotherapuetics.

Herein we set out to determine the expression and function of P-gp in a preclinical model of brain metastases of breast cancer using quantitative fluorescence microscopy and autoradiography. We observed that P-gp is expressed at the BTB in brain metastases at nearly similar levels to the BBB. In addition, P-gp is highly functional in limiting the lesion accumulation of the P-gp substrate, Rhodamine 123 (R123) despite significant passive permeability increases.

\section{MATERIALS AND METHODS CHEMICALS}

R123 was purchased from Molecular Probes Invitrogen (Eugene, OR, USA). Verapamil was purchased from Sigma (St. Louis, MO, USA). Cyclosporine A was purchased from Tocris Biochemicals (St. Louis, MO, USA). ${ }^{14}$ C-labeled aminoisobutyric acid (AIB) was purchased from American Radiolabelled Chemicals (St. Louis, MO, USA). All other chemicals used were of analytical grade and were used as supplied.

\section{ANIMALS}

Female NuNu mice ( $\sim 24$ g; 8 weeks of age) were purchased from Charles River Laboratories (Kingston, NY, USA) and were used for all the perfusion experiments done in this study. All studies were approved by the Animal Care and Use Committee and were performed in accordance with the NIH Guidelines for the Care and Use of Laboratory Animals.

\section{IN SITU MOUSE HEART PERFUSION TECHNIQUE}

The in situ mouse heart perfusion technique was utilized to evaluate brain uptake of R123 (Takasato et al., 1984; Lockman et al., 2003a) Mice were anesthetized with ketamine/xylazine (100 and $8 \mathrm{mg} / \mathrm{kg}$, respectively) and the heart exposed. Body temperature was monitored and maintained at $37^{\circ} \mathrm{C}$ using a heating pad attached to a feedback device (YSI Indicating Controller, Yellow Springs, OH, USA). Prior to insertion of the cannula, the right cardiac atrium was cut to prevent venous return. Cannulation of the left cardiac ventricle was done using butterfly syringe (28G) attached to a perfusion apparatus. Perfusion fluid was pumped into the left cardiac ventricle by a cannula at a constant rate of $2.5 \mathrm{~mL} / \mathrm{min}$ (Dagenais etal., 2000) using a Harvard Model 944 dual channel pump (Harvard Apparatus, South Natick, MA).

The perfusion fluid consisted of $\mathrm{HCO}_{3}$ buffered physiological saline, containing $128 \mathrm{mM} \mathrm{NaCl}, 24 \mathrm{mM} \mathrm{NaHCO}_{3}, 4.2 \mathrm{mM} \mathrm{KCl}$, $2.4 \mathrm{mM} \mathrm{NaH}_{2} \mathrm{PO}_{4}, 1.5 \mathrm{mM} \mathrm{CaCl}_{2}, 0.9 \mathrm{mM} \mathrm{MgSO}_{4}$, and $9 \mathrm{mM}$ glucose $(\mathrm{pH} \sim 7.35 ;[\mathrm{Na}]=154.4 \mathrm{mM})$. All solutions were filtered, oxygenated, warmed to $37^{\circ} \mathrm{C}$, and adjusted to $\mathrm{pH} 7.35$ prior to perfusion. To determine initial brain uptake of R123, perfusion fluid containing R123 (50 $\mu \mathrm{g} / \mathrm{mL})$ was infused into the systemic circulation for 30-120 s. At the end of each experiment, mice were sacrificed, and the brain was rapidly removed $(<60 \mathrm{~s})$ from the skull. The brain was flash frozen in isopentane $\left(-65^{\circ} \mathrm{C}\right)$. Concentration of the fluorophore (R123) in brain was determined using fluorescent microscopy and regional permeability was expressed by the unidirectional transfer constants, $\mathrm{K}_{\mathrm{in}}(\mathrm{mL} / \mathrm{s} / \mathrm{g})$ derived from Eq. 1.

\section{QUANTIFICATION OF R123 USING FLUORESCENCE MICROSCOPY}

Fluorescence was observed with an Olympus MVX10 stereomicroscope (objective: $2 \times$, NA 0.5 ) with an optical zoom range from 0.63 to 12.6 . The excitation and emission of R123 was obtained using a GFP filter (excitation/band pass filter of 470/40, emission/band pass filter of 525/50 and dichromatic mirror at $495 \mathrm{~nm}$; Chroma Technology, Bellow Falls, VT, USA). Tissue sections of $20 \mu \mathrm{m}$ were obtained at $-23^{\circ} \mathrm{C}$ using a cryotome (Leica CM3050S, Leica Microsystems, Buffalo Grove, IL, USA), mounted on charged glass slides, and kept at $-23^{\circ} \mathrm{C}$. Data were analyzed using quantitative fluorescence microscopy and all images were obtained with $15 \mathrm{~ms}$ exposures, though a 2.0 objective at $4 \times$ magnification (Olympus MVX10) with a monochromatic cooled CCD scientific camera (Retiga 4000R, QImaging, Surrey, BC, Canada). Slidebook ${ }^{\circledR} 5$ software (Intelligent Imaging Innovations, Denver, CO, USA) was utilized to determine sum intensity per gram of brain which then converted into concentration of dye per gram of brain using the brain homogenate standards. The voxel by voxel sum intensity of fluorescence for brain homogenate samples was obtained with the $2 \times$ objective. The optical zoom range was maintained at $4 \times$ for a total optical magnification of $8 \times$. The sum intensity per gram of brain homogenate was obtained using a set exposure time of $15 \mathrm{~ms}$ with camera gain settings of 615 . The total fluorescence intensity signal for each concentration was then plotted as a function of grams of brain which was calculated using the area in microns squared multiplied by the thickness of the brain sample $(20 \mu \mathrm{m})$ to obtain a total brain volume that was analyzed. The brain volume $\left(\mu \mathrm{m}^{3}\right)$ was multiplied by the density of brain tissue $\left(1.04 \mathrm{~g} / \mathrm{cm}^{3}\right)$ as similarly reported by (Tengvar et al., 1983) to obtain a weight of brain tissue that was analyzed.

\section{PREPARATION OF BRAIN STANDARDS}

To calculate the concentration of the R123 in brain, standard curves were generated in rat brain homogenates. Briefly, $100 \mu \mathrm{L}$ of standard solution of the dye was added to each of $500 \mathrm{mg}$ of the brain and homogenized. The homogenized mass was flash frozen 
in isopentane $\left(-80^{\circ} \mathrm{C}\right)$ and sliced into $20 \mu \mathrm{m}$ sections using a cryostat $-23^{\circ} \mathrm{C}$ and mounted onto glass, superfrost slides. The slices were analyzed using quantitative fluorescence microscopy and the sum intensity per gram of brain homogenate was plotted against concentration of the dye.

\section{KINETIC ANALYSIS}

Unidirectional uptake transfer constants $\left(K_{\text {in }}\right)$ were calculated from the following relationship to the linear portion of the uptake curve:

$$
Q^{*} / C^{*}=K_{\text {in }} T+V_{0}
$$

where $Q^{*}$ is the quantity of fluorophore $(\mathrm{R} 123)$ in brain $(\mu \mathrm{g} / \mathrm{g})$ at the end of perfusion, $C^{\star}$ is the perfusion fluid concentration of fluorophore $(\mu \mathrm{g} / \mathrm{mL}), T$ is the perfusion time $(\mathrm{s})$ and $V_{0}$ is the extrapolated intercept ( $T=0 \mathrm{~s}$; "vascular volume" in $\mathrm{mL} / \mathrm{g}$ ). After determination of a perfusion time that allowed adequate amount of fluorescent marker to pass into brain and yet remained in the linear uptake zone, $K_{\text {in }}$ was determined in single time-point experiments as:

$$
\begin{aligned}
K_{\text {in }}= & {\left.\left[Q^{*}-V_{0} C^{*}\right] / C^{*} T\right] } \\
& (\text { Takasato et al., 1984; Smith and Takasato, 1986). }
\end{aligned}
$$

\section{ANTIBODY STAINING}

Tissues were rehydrated in PBS and then fixed in $4 \%$ paraformaldehyde (PFA) for P-gp (Abcam, Cambridge, MA), cytokeratin (Abcam) and CD31 (BD Pharmingen, San Jose, CA), ice-cold methanol for ABCB1 (Santa Cruz Biotechnology), CD31 (BD Pharmingen). After three PBS washings (5 min), slides were blocked with $4 \%$ goat serum and $0.2 \%$ Triton-X 100 (1 h). After blocking, primary antibodies were added, followed by overnight incubation at $4^{\circ} \mathrm{C}$. The next day, the slides were washed and secondary antibodies and DAPI $(1 \mathrm{mg} / \mathrm{mL})$ were added $(1 \mathrm{~h})$. Slides were again washed, DAKO mounting medium was added, and coverslips were applied.

\section{RESULTS}

To determine if P-gp expression is present in the vasculature of brain metastases, we analyzed the brains of tumor bearing mice using immunofluorescence staining for both P-gp and the vascular marker CD31 to quantify the amount of colocalization (Figure 1). There was significant expression of P-gp at the $\mathrm{BBB}$ and $\mathrm{BTB}$ (Figure 1B). Overall there was no difference between the fluorescent intensity of P-gp staining in the CD-31 defined regions in tumor vasculature $(22.9 \pm 0.4$ A.U.; $n=756$ vessels) and in the normal brain vasculature $(22.6 \pm 0.3$ A.U.; $n=1214$ ). In addition, there was positive P-gp staining that did not co-localize to the vasculature, but surrounded metastasis cells suggesting that $\mathrm{P}$-gp may also be present on the metastatic cancer cells.

We measured P-gp function by the time dependent accumulation of the fluorescence P-gp substrate R123 according to previous methodology (Mittapalli et al., 2013). Using fluorescent brain standards we determined the blood to brain unidirectional transfer coefficient $\left(K_{\text {in }}\right)$ of R123 in normal brain and in metastatic lesions by calculating the concentration of R123 divided by the concentration in the perfusate and plotted this over time (30-120 s; Figure 2A). We then applied a previously calculated correction to the vascular volume by perfusion of non-permeable $\left[{ }^{14} \mathrm{C}\right]-$ sucrose and measuring its vascular space $(0.015 \pm 0.002 \mathrm{~mL} / \mathrm{g})$. We observed that the uptake of R123 was linear within the perfusion time with a $K_{\text {in }}$ of $0.12 \pm 0.03 \mu \mathrm{L} / \mathrm{s} / \mathrm{g}$. To determine if we could inhibit P-gp mediated efflux of R123, we added P-gp inhibitors verapamil and cyclosporine A (Choi and Li, 2005; Breedveld et al., 2006; Baumert and Hilgeroth, 2009) at various concentrations to the R123 perfusate in separate experiments (Figure 2A). Upon co-perfusion of R123 and each inhibitor, there was an increase in R123 permeability; Cyclosporin A $(2.4 \pm 0.5 \mu \mathrm{L} / \mathrm{s} / \mathrm{g})$; and Verapamil $(2.2 \pm 0.2 \mu \mathrm{L} / \mathrm{s} / \mathrm{g})]$ indicating that R123 uptake into brain is limited by the efflux function of P-gp at the BBB.

We then plotted R123's LogD (octanol/water coefficient; $\mathrm{pH}=7.4)$ and observed $K_{\mathrm{in}}$ in comparison to known passive

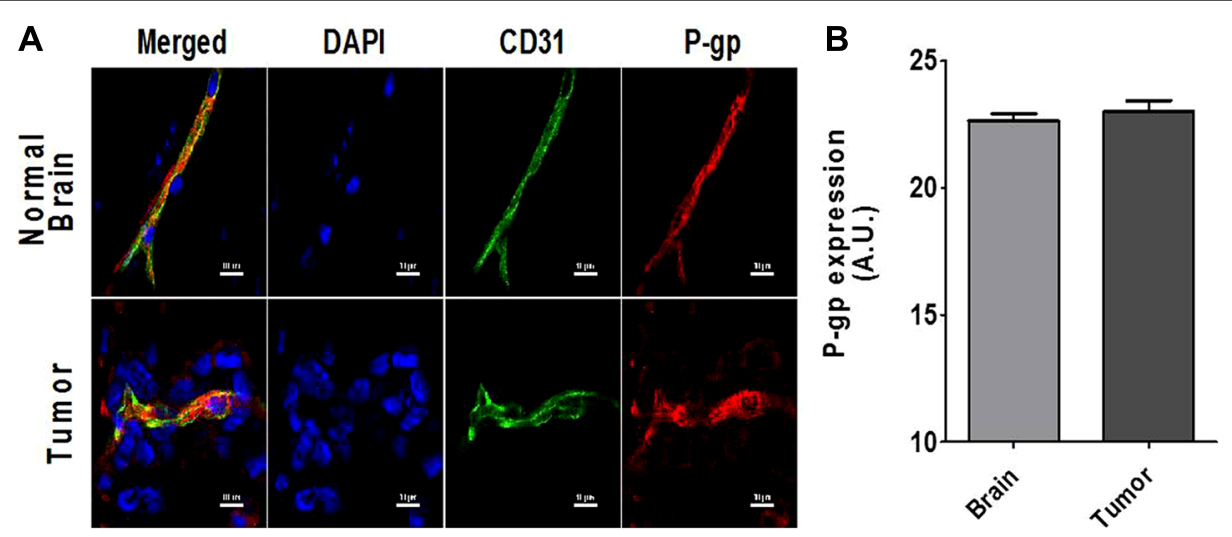

FIGURE 1 | (A) Representative images of the co-localized expression of P-gp (red) in a capillary in normal brain (top row) and a blood vessel in a $231 \mathrm{Br}$ brain metastasis (bottom row) are shown. Endothelial nuclei as well as the nuclei of the 231 $\mathrm{Br}$ lesions are shown in blue (DAPI). Blood vessels (CD-31 expression) in both sections are shown in green (Alexa Fluor 488). P-gp expression is shown in red (Alexa Fluor 594). (B) The bar graph shows the relative P-gp expression per vessel as defined by CD31 stained regions. Mean $+\mathrm{SEM}$; $\mathrm{BTB} ; n=756$ vessels and $\mathrm{BBB} n=1214$ vessels) 

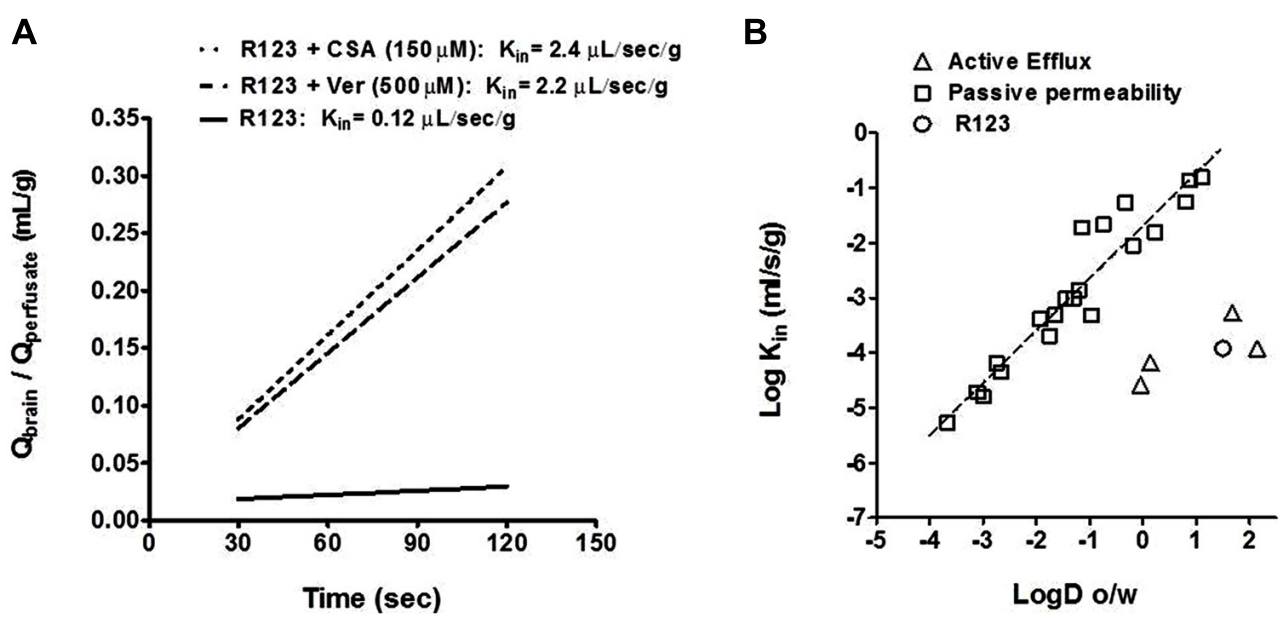

FIGURE 2 | (A) The $K_{\text {in }}$ of R123 in the presence of the known P-gp inhibitors, cyclosporin $A$ and verapamil, increases brain distribution (as reported by the $K_{\text {in }}$ ) by $>10$-fold. All data represent mean \pm S.E.M for total brain; $n=3-5$ for all groups. Statistics: one-way ANOVA; Dunnett's. (B) The relationship

between LogD (octanol/water coefficient; $\mathrm{pH}=7.4$ ) and observed $K_{\text {in }}$ is used to profile a molecule or drug's mechanism of distribution into brain. Compounds that are known to cross the BBB via passive diffusion are plotted using gray squares and those subject to efflux are plotted with gray triangles. R123 (open circle) falls below $\sim 3$ log units the line of identity for passive permeability indicating it may be subject to efflux. permeability compounds (Begley, 1996) and efflux substrates (Summerfield et al., 2007; Figure 2B). Molecules and drugs that passively diffuse into brain exhibit a linear relationship between their $\log \mathrm{D}$ (octanol/water coefficient) and their observed Log $K_{\text {in }}$ while molecules which are subject to efflux will exhibit observed $\log K_{\text {in }}$ values well below the value predicted by its $\log \mathrm{D}$ (Figure 2B). R123 has a $\operatorname{LogD}$ of 1.51 (Forster et al., 2012) and $\log K_{\text {in }}$ of -3.93 (calculated from observed $K_{\text {in }}$; Figure 2A) which places R123 several orders of magnitude below a passively diffusing molecule's profile which supports the evidence of R213's restriction from brain via an efflux transporter.

To determine BTB passive permeability and whether P-gp influences R123 uptake into brain metastases of breast cancer, tumor-bearing mice were injected with ${ }^{14} \mathrm{C}$-AIB (passive permeability tracer) which was allowed to circulate for $10 \mathrm{~min}$ before a 2 min R123 perfusion, which was followed by sacrifice (Figure 3). Autoradiography analysis of the brains revealed elevated permeability to ${ }^{14} \mathrm{C}$-AIB $(\sim 4.9$-fold increase). The passive permeability marker tracer's uptake did not correlate $\left(r^{2}=0.17\right.$ for AIB) with metastases size (Figure 3A). R123 uptake, however, was not different from that of normal brain on average $(\sim 0.98$-fold change) in metastatic lesions and had no correlation $\left(r^{2}=0.033\right)$ to metastasis size (Figure 3B). R123 permeability did not correlate passive permeability changes as measured by ${ }^{14} \mathrm{C}$-AIB $\left(r^{2}=0.0008\right.$ ) (Figure $3 \mathrm{C}$ ) accumulation suggesting that $\mathrm{R} 123$ remains restricted from the brain parenchyma via P-gp mediated efflux. The observed R123 $K_{\text {in }}$ value for normal brain (BDT; brain distant to tumor) regions of metastases bearing mice $\left(K_{\text {in }}=0.11 \pm 0.06 \times 10^{-1} \mu \mathrm{L} / \mathrm{s} / \mathrm{g}\right.$ ) (Figures $4 \mathrm{~A}-\mathrm{C}$ ) was consistent with previous $K_{\text {in }}$ measurements in tumor-free mice (Figure 1B). And, the $K_{\text {in }}$ of R123 in the BTB (within metastases) was $0.12 \pm 0.003 \mu \mathrm{L} / \mathrm{s} / \mathrm{g}$ which was not different than that of normal brain $(p>0.05)$.

\section{DISCUSSION AND CONCLUSION}

In the current study, we present data suggesting P-gp retains its efflux function at the BTB despite a disruption in the integrity of the $\mathrm{BBB}$ induced by the presence of a metastatic lesion. Of notable methodology, to the best of our knowledge we are the first to combine quantitative fluorescence microscopy to measure R123 P-gp mediated efflux and quantitative autoradiography to measure changes in BTB passive permeability $\left({ }^{14} \mathrm{C}\right.$-AIB $)$ in the same brain slice. This method is able to directly shed light on two independent processes occurring at the BTB.

The utilization of R123 to evaluate P-gp function is well established (Hegmann et al., 1992). However, there is less evidence regarding R123's affinity and efflux transport to other transporters that contribute to drug restriction to brain. R123 has been reported to be subject to transport by BCRP (Doyle et al., 1998), and OCT 1 \& 2 (Jouan et al., 2012), and MRP2 (Munic et al., 2011). Though studies using specific transporter inhibitors at correct concentrations show P-gp primarily transports R123 and restricts accumulation into brain (Wang et al., 1995). Moreover, the magnitude of R123 efflux by P-gp is greater than that of BCRP and MRP1 (Chopra, 2004) and therefore should represent the major pathway of active efflux transport at the $\mathrm{BBB}$ and BTB.

Due to the difficulty in performing the in situ brain perfusion in mice, we modified the in situ brain perfusion to a cardiac perfusion method in female $\mathrm{Nu} / \mathrm{Nu}$ mice bearing brain metastases of breast cancer to characterize P-gp function in vivo. This method has similar advantages to the in situ brain perfusion method in that we may control aspects of the perfusion to determine both influx and efflux kinetics, transporter inhibition coefficients, and BTB or BBB permeability (Smith and Allen, 2003). This control helps determine accurate apparent permeability coefficients (Lockman et al., 2005a), the degree to what a substrate is efflux back into blood (Lockman et al., 2003b), inhibition constants for 

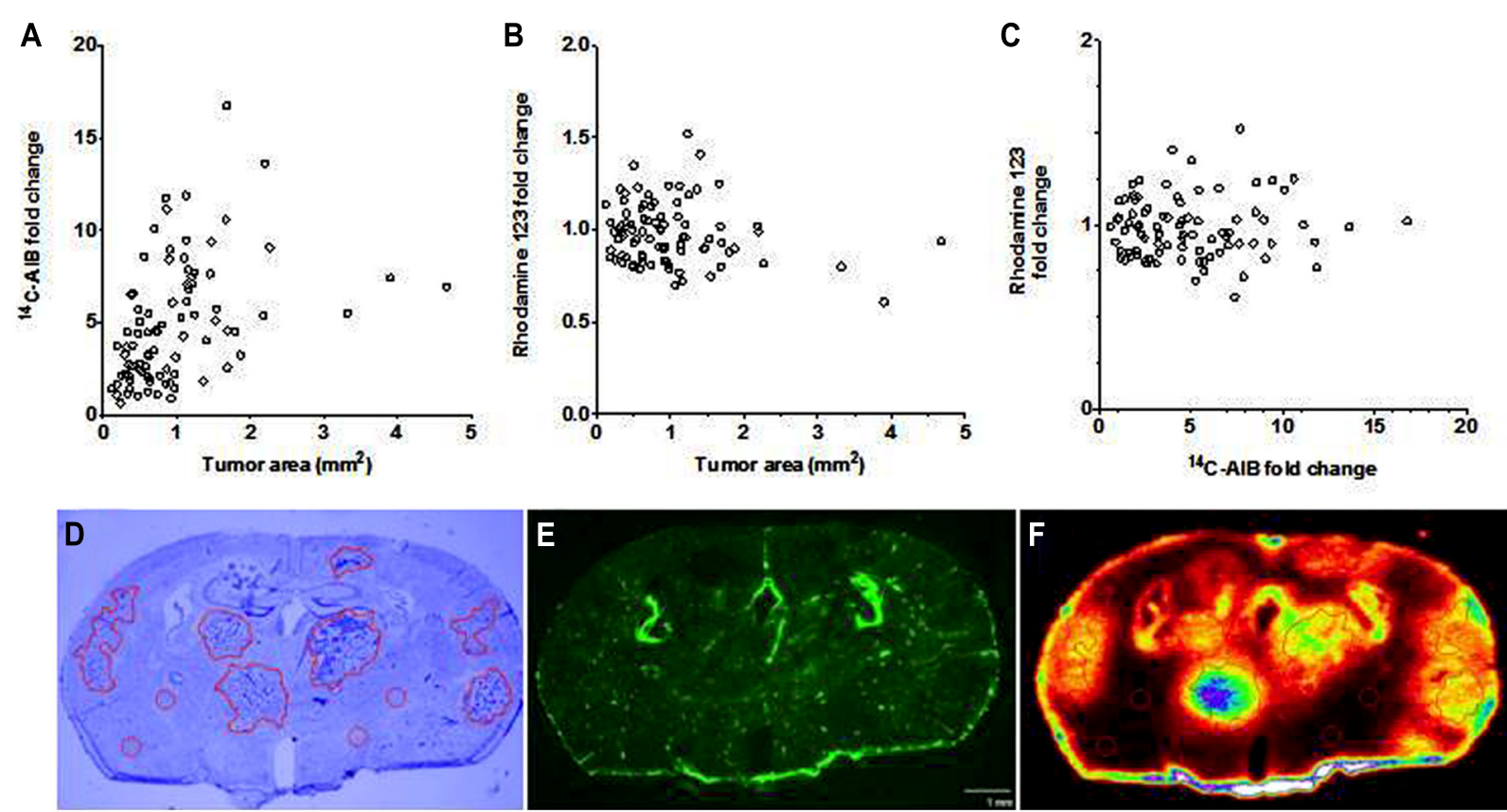

FIGURE 3 | The BTB is variably compromised for compounds entering via passive diffusion but retains P-gp mediated efflux. The passive

permeability marker ${ }^{14} \mathrm{C}$-AIB fold change in brain metastases did not correlate $\left(r^{2}=0.167\right)$ with metastasis size $\left(\mathrm{mm}^{2}\right)$ (A). R123 fold change in brain metastases did not correlate $\left(r^{2}=0.033\right)$ with metastasis size (B). There was no observed relationship $\left(r^{2}=0.0009\right)$ between the fold increase in brain metastases of ${ }^{14} \mathrm{C}$-AIB (passive permeability marker) and R123 (P-gp substrate) (C). One representative brain slice showing metastases location (D, cresyl violet), R123 fluorescence distribution ( $\mathbf{E}$, fluorescence microscopy), and ${ }^{14} \mathrm{C}$-AlB brain uptake (F, quantitative autoradiography).

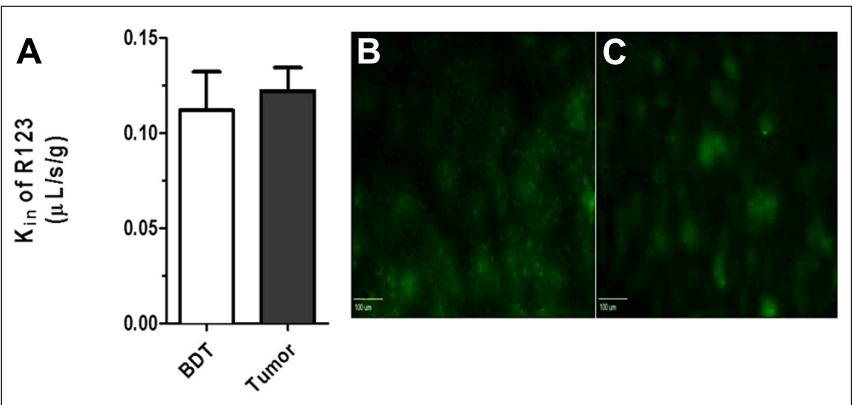

FIGURE 4 | (A) No difference ( $p>0.05$; student $t$-test, $n=3-5$ ) was seen between $\mathrm{R} 123$ blood to brain transfer constant $K_{\text {in }}$ between values measured in normal brain $\left(K_{\text {in }}=0.11 \pm 0.01 \mu \mathrm{L} / \mathrm{s} / \mathrm{g}\right)$ and metastases $\left(K_{\text {in }}=0.12 \pm 0.02 \mu \mathrm{L} / \mathrm{s} / \mathrm{g}\right)$. Representative R123 fluorescence images in normal brain (B) and within a metastasis (C) (scale bar $=100 \mu \mathrm{m})$.

transporters (Lockman et al., 2001) and a direct measurement of BBB and BTB integrity (Lockman et al., 2003a, 2004, 2005b)

Using the cardiac perfusion method, R123 accumulated in brain linearly over 2 min of perfusion time. Our observed blood to brain transfer constant $\left(K_{\text {in }}\right)$ was $\sim 10$-fold less than what would be the calculated $K_{\text {in }}$ based on values of similar molecules in terms of their octanol/water coefficient and molecular weight. The lower observed $K_{\text {in }}$, is typically seen when the compound is actively extruded by the BBB back into blood (Begley, 1996). Further confirmation that R123 is extruded by an efflux mechanism at the BBB was suggested by the significantly increased uptake of R123 from blood to brain after the addition of verapamil or cyclosporine A to the perfusate (Mittapalli et al., 2013).

Of importance to this study, the simultaneous administration of a passive permeability marker and a tracer subject to P-gp mediated efflux allowed us to measure BTB integrity and functional efflux. Both parameters have been shown to significantly impact drug uptake into metastases (Lockman et al., 2010) but have not been simultaneously measured directly in metastatic lesions. Our initial hypothesis prior to the experiment was that since we have seen increased permeability at the BTB in metastases (Lockman et al., 2010), we would also see a similar increase in R123 distribution into the lesion. However we did not observe R123 accumulation within metastases.

There are two possible explanations that may provide insight to the lack of increased R123 permeability in the lesion. First, it is known that P-gp is expressed in the vasculature of human brain tumors and metastases (Guo et al., 2010). Although, P-gp expression at the BTB has been shown to be variable among different types of tumors within the CNS (Cordon-Cardo et al., 1990; Toth et al., 1996; Tews etal., 2000) as well as different between separate intracranial metastases (Demeule et al., 2001; Lockman et al., 2010). We observed some variability of P-gp expression in the vessels of our metastases, but overall P-gp expression was not significantly different in the over 2,000 vessels we analyzed between the BTB and the BBB. Accordingly, this may be a reason why there was little overall difference in tissue accumulation of R123 between the two tissue types. Another possible explanation is that we observed tumor cells directly adjacent or proximal to 
the vasculature also express P-gp, which may also contribute to the restriction of R123 in the lesions. Overall, the pattern of distribution for each tracer suggests that the BTB is disrupted yet its efflux transport mechanisms are intact and can limit brain and or tumor uptake of P-gp substrates.

This work does have translational value to human drug distribution to brain. The expression of BCRP at human BBB is $\sim 2$ fold higher as compared to the expression levels at mouse BBB. The $\mathrm{P}$-gp expression is 3 fold higher at mouse $\mathrm{BBB}$ as compared to the expression levels at human BBB. So BCRP still plays a major role at human $\mathrm{BBB}$ (Hoshi et al., 2013) suggesting P-gp plays a major functional role in the human $\mathrm{BBB}$. While some studies have supported little efflux contribution for various anti-cancer drug to brain (Agarwal et al., 2011), others have demonstrated P$\mathrm{gp}$ at the BBB and BTB restricts the uptake of many anti-cancer agents; such as paclitaxel, docetaxel, vemurafenib, erlotinib, axitinib, and tamoxifen (Gallo et al., 2003; Kemper et al., 2004; Wang et al., 2010; Iusuf et al., 2011; Poller et al., 2011; Mittapalli et al., 2012; Taskar et al., 2012; Agarwal et al., 2013). Attempts to modify P-gp using inhibitors have shown promise in preclinical settings (Kemper et al., 2004; Mittapalli et al., 2012; Agarwal et al., 2013).

Although we, and others, have observed variably elevated accumulations of small molecules across the BTB in brain metastases, the data herein provide evidence that $\mathrm{P}$-gp retains much of its residual function. Thus, BTB function in this preclinical model may be viewed as only partially compromised and retains significant ability to impede uptake of therapeutic compounds. Given the large list of drugs, particularly anticancer agents such as paclitaxel and doxorubicin, which are subject to P-gp mediated efflux, the clinical impact of this retained function suggests the BTB remains a significant barrier in delivering chemotherapeutics into metastatic lesions.

\section{AUTHORS' CONTRIBUTIONS}

1. Concept and design - Chris E. Adkins, Rajendar K. Mittapalli, Vamshi K. Manda, Paul R. Lockman

2. Development of methodology Chris E. Adkins, Rajendar K. Mittapalli, Vamshi K. Manda, Kaci A. Bohn, Paul R. Lockman

3. Acquisition of data (provided animals, acquired and managed patients, provided facilities, etc) Chris E. Adkins, Rajendar K. Mittapalli, Vamshi K. Manda, Tori B. Terrell, Kaci A. Bohn, Celik Yasemin, Tiffany R. Grothe, Julie A. Lockman, Paul R. Lockman

4. Analysis and interpretation of data (e.g., statistical analysis, biostatistics, computational analysis) Chris E. Adkins, Rajendar K. Mittapalli, Vamshi K. Manda, Mohamed I. Nounou, Afroz S. Mohammad, Tori B. Terrell, Julie A. Lockman, Paul R. Lockman

5. Writing, review, and/or revision of the manuscript Chris E. Adkins, Mohamed I. Nounou, Afroz S. Mohammad, Tori B. Terrell, Paul R. Lockman

6. Study supervision Paul R. Lockman

\section{ACKNOWLEDGMENT}

Funding for this study was provided by the Department of Defense Breast Cancer Research Program Grant W81 XWH-062-0033 and National Cancer Institute, National Institutes of Health, Bethesda, MD. 1R01CA166067-01A1 to Paul R. Lockman.

\section{REFERENCES}

Abbott, N. J., Patabendige, A. A., Dolman, D. E., Yusof, S. R., and Begley, D. J. (2010). Structure and function of the blood-brain barrier. Neurobiol. Dis. 37, 13-25. doi: 10.1016/j.nbd.2009.07.030

Agarwal, S., Hartz, A. M., Elmquist, W. F., and Bauer, B. (2011). Breast cancer resistance protein and P-glycoprotein in brain cancer: two gatekeepers team up. Curr. Pharm. Des. 17, 2793-2802. doi: 10.2174/138161211797 440186

Agarwal, S., Manchanda, P., Vogelbaum, M. A., Ohlfest, J. R., and Elmquist, W. F. (2013). Function of the blood-brain barrier and restriction of drug delivery to invasive glioma cells: findings in an orthotopic rat xenograft model of glioma. Drug Metab. Dispos. 41, 33-39. doi: 10.1124/dmd.112.048322

Baumert, C., and Hilgeroth, A. (2009). Recent advances in the development of P-gp inhibitors. Anticancer Agents Med. Chem. 9, 415-436. doi: 10.2174/1871520610909040415

Begley, D. J. (1996). The blood-brain barrier: principles for targeting peptides and drugs to the central nervous system. J. Pharm. Pharmacol. 48, 136-146. doi: 10.1111/j.2042-7158.1996.tb07112.x

Breedveld, P., Beijnen, J. H., and Schellens, J. H. (2006). Use of P-glycoprotein and BCRP inhibitors to improve oral bioavailability and CNS penetration of anticancer drugs. Trends Pharmacol. Sci. 27, 17-24. doi: 10.1016/j.tips.2005.11.009

Breedveld, P., Pluim, D., Cipriani, G., Wielinga, P., van Tellingen, O., Schinkel, A. H., et al. (2005). The effect of Bcrpl (Abcg2) on the in vivo pharmacokinetics and brain penetration of imatinib mesylate (Gleevec): implications for the use of breast cancer resistance protein and P-glycoprotein inhibitors to enable the brain penetration of imatinib in patients. Cancer Res. 65, 2577-2582. doi: 10.1158/0008-5472.CAN-04-2416

Choi, J. S., and Li, X. (2005). The effect of verapamil on the pharmacokinetics of paclitaxel in rats. Eur. J. Pharm. Sci. 24, 95-100. doi: 10.1016/j.ejps.2004.10.002

Chopra, A. (2004). "11C-Labeled rhodamine-123," in Molecular Imaging and Contrast Agent Database (MICAD) (Bethesda:National Center for Biotechnology Information).

Cordon-Cardo, C., O’Brien, J. P., Boccia, J., Casals, D., Bertino, J. R., and Melamed, M. R. (1990). Expression of the multidrug resistance gene product (P-glycoprotein) in human normal and tumor tissues. J. Histochem. Cytochem. 38, 1277-1287. doi: 10.1177/38.9.1974900

Dagenais, C., Rousselle, C., Pollack, G. M., and Scherrmann, J. M. (2000). Development of an in situ mouse brain perfusion model and its application to mdr1a P-glycoprotein-deficient mice. J. Cereb. Blood Flow Metab. 20, 381-386. doi: 10.1097/00004647-200002000-00020

Deeken, J. F., and Loscher, W. (2007). The blood-brain barrier and cancer: transporters, treatment, and Trojan horses. Clin. Cancer Res. 13, 1663-1674. doi: 10.1158/1078-0432.CCR-06-2854

Demeule, M., Shedid, D., Beaulieu, E., Del Maestro, R. F., Moghrabi, A., Ghosn, P. B., et al. (2001). Expression of multidrug-resistance P-glycoprotein (MDR1) in human brain tumors. Int. J. Cancer 93, 62-66. doi: 10.1002/ijc.1306

Doyle, L. A., Yang, W., Abruzzo, L. V., Krogmann, T., Gao, Y., Rishi, A. K., et al. (1998). A multidrug resistance transporter from human MCF-7 breast cancer cells. Proc. Natl. Acad. Sci. U.S.A. 95, 15665-15670. doi: 10.1073/pnas.95.26.15665

Forster, S., Thumser, A. E., Hood, S. R., and Plant, N. (2012). Characterization of rhodamine-123 as a tracer dye for use in in vitro drug transport assays. PLoS ONE 7:e33253. doi: 10.1371/journal.pone.0033253

Gallo, J. M., Li, S., Guo, P., Reed, K., and Ma, J. (2003). The effect of P-glycoprotein on paclitaxel brain and brain tumor distribution in mice. Cancer Res. 63, 5114-5117.

Guo, Z., Zhu, J., Zhao, L., Luo, Q., and Jin, X. (2010). Expression and clinical significance of multidrug resistance proteins in brain tumors. J. Exp. Clin. Cancer Res. 29, 122. doi: 10.1186/1756-9966-29-122

Hagenbuch, B., and Meier, P. J. (2004). Organic anion transporting polypeptides of the OATP/ SLC21 family: phylogenetic classification as OATP/ SLCO superfamily, new nomenclature and molecular/functional properties. Pflugers Arch. 447, 653665. doi: 10.1007/s00424-003-1168-y

Hawkins, B. T., and Davis, T. P. (2005). The blood-brain barrier/neurovascular unit in health and disease. Pharmacol. Rev. 57, 173-185. doi: 10.1124/pr.57.2.4

Hegmann, E. J., Bauer, H. C., and Kerbel, R. S. (1992). Expression and functional activity of P-glycoprotein in cultured cerebral capillary endothelial cells. Cancer Res. 52, 6969-6975.

Henson, J. W., Cordon-Cardo, C., and Posner, J. B. (1992). P-glycoprotein expression in brain tumors. J. Neurooncol. 14, 37-43. doi: 10.1007/BF00170943 
Hoshi, Y., Uchida, Y., Tachikawa, M., Inoue, T., Ohtsuki, S., and Terasaki, T. (2013). Quantitative atlas of blood-brain barrier transporters, receptors, and tight junction proteins in rats and common marmoset. J. Pharm. Sci.102, 3343-3355. doi: 10.1002/jps.23575

Iusuf, D., Teunissen, S. F., Wagenaar, E., Rosing, H., Beijnen, J. H., and Schinkel, A. H. (2011). P-glycoprotein (ABCB1) transports the primary active tamoxifen metabolites endoxifen and 4-hydroxytamoxifen and restricts their brain penetration. J. Pharmacol. Exp. Ther. 337, 710-717. doi: 10.1124/jpet.110. 178301

Jouan, E., Le Vee, M., Denizot, C., Da Violante, G., and Fardel, O. (2012). The mitochondrial fluorescent dye rhodamine 123 is a high-affinity substrate for organic cation transporters (OCTs) 1 and 2. Fundam. Clin. Pharmacol. doi: 10.1111/j.1472-8206.2012.01071.x [Epub ahead of print].

Kemper, E. M., Verheij, M., Boogerd, W., Beijnen, J. H., and van Tellingen, O. (2004). Improved penetration of docetaxel into the brain by coadministration of inhibitors of P-glycoprotein. Eur. J. Cancer 40, 1269-1274. doi: 10.1016/j.ejca.2004.01.024

Lipinski, C. A., Lombardo, F., Dominy, B. W., and Feeney, P. J. (2001). Experimental and computational approaches to estimate solubility and permeability in drug discovery and development settings. Adv. Drug Deliv. Rev. 46, 3-26. doi: 10.1016/S0169-409X(00)00129-0

Lockman, P. R., Koziara, J. M., Mumper, R. J., and Allen, D. D. (2004). Nanoparticle surface charges alter blood-brain barrier integrity and permeability. J. Drug Target 12, 635-641. doi: 10.1080/10611860400015936

Lockman, P. R., Koziara, J., Roder, K. E., Paulson, J., Abbruscato, T. J., Mumper, R. J.,et al. (2003a). In vivo and in vitro assessment of baseline blood-brain barrier parameters in the presence of novel nanoparticles. Pharm. Res. 20, 705-713. doi: 10.1023/A:1023492015851

Lockman, P. R., Mumper, R. J., and Allen, D. D. (2003b). Evaluation of blood-brain barrier thiamine efflux using the in situ rat brain perfusion method. J. Neurochem. 86, 627-634. doi: 10.1046/j.1471-4159.2003. 01888.x

Lockman, P. R., McAfee, G., Geldenhuys, W. J., Van der Schyf, C. J., Abbruscato, T. J., and Allen, D. D. (2005a). Brain uptake kinetics of nicotine and cotinine after chronic nicotine exposure. J. Pharmacol. Exp. Ther. 314, 636-642. doi: 10.1124/jpet.105.085381

Lockman, P. R., Van der Schyf, C. J., Abbruscato, T. J., and Allen, D. D. (2005b). Chronic nicotine exposure alters blood-brain barrier permeability and diminishes brain uptake of methyllycaconitine. J. Neurochem. 94, 37-44. doi: 10.1111/j.14714159.2005.03162.x

Lockman, P. R., Mittapalli, R. K., Taskar, K. S., Rudraraju, V., Gril, B., Bohn, K. A. et al. (2010). Heterogeneous blood-tumor barrier permeability determines drug efficacy in experimental brain metastases of breast cancer. Clin. Cancer Res. 16, 5664-5678. doi: 10.1158/1078-0432.CCR-10-1564

Lockman, P. R., Roder, K. E., and Allen, D. D. (2001). Inhibition of the rat bloodbrain barrier choline transporter by manganese chloride. J. Neurochem. 79, 588 594. doi: 10.1046/j.1471-4159.2001.00589.x

Loscher, W., and Potschka, H. (2005). Role of drug efflux transporters in the brain for drug disposition and treatment of brain diseases. Prog. Neurobiol. 76, 22-76. doi: 10.1016/j.pneurobio.2005.04.006

Mittapalli, R. K., Manda, V. K., Bohn, K. A., Adkins, C. E., and Lockman, P. R. (2013). Quantitative fluorescence microscopy provides high resolution imaging of passive diffusion and P-gp mediated efflux at the in vivo bloodbrain barrier. J. Neurosci. Methods 219, 188-195. doi: 10.1016/j.jneumeth.2013. 07.001

Mittapalli, R. K., Vaidhyanathan, S., Sane, R., and Elmquist, W. F. (2012). Impact of P-glycoprotein $(\mathrm{ABCB} 1)$ and breast cancer resistance protein (ABCG2) on the brain distribution of a novel BRAF inhibitor: vemurafenib (PLX4032). J. Pharmacol. Exp. Ther. 342, 33-40. doi: 10.1124/jpet.112. 192195

Munic, V., Hlevnjak, M., and Erakovic Haber, V. (2011). Characterization of rhodamine-123, calcein and 5(6)-carboxy-2',7'-dichlorofluorescein (CDCF) export via MRP2 (ABCC2) in MES-SA and A549 cells. Eur. J. Pharm. Sci. 43, 359-369. doi: 10.1016/j.ejps.2011.05.003

Pardridge, W. M. (2007). Blood-brain barrier delivery. Drug Discov. Today, 12, 54-61. doi: 10.1016/j.drudis.2006.10.013

Poller, B., Iusuf, D., Sparidans, R. W., Wagenaar, E., Beijnen, J. H., and Schinkel, A. H. (2011). Differential impact of P-glycoprotein (ABCB1) and breast cancer resistance protein (ABCG2) on axitinib brain accumulation and oral plasma pharmacokinetics. Drug Metab. Dispos. 39, 729-735. doi: 10.1124/dmd.110.037317

Polli, J. W., Olson, K. L., Chism, J. P., John-Williams, L. S., Yeager, R. L., Woodard, S. M., etal. (2009). An unexpected synergist role of Pglycoprotein and breast cancer resistance protein on the central nervous system penetration of the tyrosine kinase inhibitor lapatinib (N-\{3-chloro-4[(3-fluorobenzyl)oxy]phenyl $\}-6-[5-(\{[2-($ methylsulfonyl)ethyl]amino $\}$ methyl)2-furyl]-4-quinazolinamine; GW572016). Drug Metab. Dispos. 37, 439-442. doi: 10.1124/dmd.108.024646

Schinkel, A. H., Wagenaar, E., Mol, C. A., and van Deemter, L. (1996). P-glycoprotein in the blood-brain barrier of mice influences the brain penetration and pharmacological activity of many drugs. J. Clin. Invest. 97, 2517-2524. doi: 10.1172/JCI118699

Schinkel, A. H., Wagenaar, E., van Deemter, L., Mol, C. A., and Borst, P. (1995). Absence of the mdrla P-Glycoprotein in mice affects tissue distribution and pharmacokinetics of dexamethasone, digoxin, and cyclosporin A. J. Clin. Invest. 96, 1698-1705. doi: 10.1172/JCI118214

Smith, Q. R., and Allen, D. D., (2003). In situ brain perfusion technique. Methods Mol. Med. 89, 209-218.

Smith, Q. R., and Takasato, Y. (1986). Kinetics of amino acid transport at the bloodbrain barrier studied using an in situ brain perfusion technique. Ann. N. Y. Acad. Sci. 481, 186-201. doi: 10.1111/j.1749-6632.1986.tb27150.x

Summerfield, S. G., Read, K., Begley, D. J., Obradovic, T., Hidalgo, I. J., Coggon, S., et al. (2007). Central nervous system drug disposition: the relationship between in situ brain permeability and brain free fraction. J. Pharmacol. Exp. Ther. 322, 205-213. doi: 10.1124/jpet.107.121525

Takasato, Y., Rapoport, S. I., and Smith, Q. R. (1984). An in situ brain perfusion technique to study cerebrovascular transport in the rat. Am. J. Physiol. 247,(Pt 2), H484-H493.

Taskar, K. S., Rudraraju, V., Mittapalli, R. K., Samala, R., Thorsheim, H. R., Lockman, J., et al. (2012). Lapatinib distribution in HER2 overexpressing experimental brain metastases of breast cancer. Pharm. Res. 29, 770-781. doi: 10.1007/s11095-0110601-8

Tengvar, C., Hultstrom, D., and Olsson, Y. (1983). An improved Percoll density gradient for measurements of experimental brain edema. Addition of sucrose to an isotonic gradient in an attempt to balance osmotic conditions during density determinations. Acta Neuropathol. 61, 201-206. doi: 10.1007/BF00691986

Tews, D. S., Nissen, A., Külgen, C., and Gaumann, A. K. (2000). Drug resistanceassociated factors in primary and secondary glioblastomas and their precursor tumors. J. Neurooncol. 50, 227-237. doi: 10.1023/A:1006491405010

Toth, K., Vaughan, M. M., Peress, N. S., Slocum, H. K., and Rustum, Y. M. (1996). MDR1 P-glycoprotein is expressed by endothelial cells of newly formed capillaries in human gliomas but is not expressed in the neovasculature of other primary tumors. Am. J. Pathol. 149, 853-858.

Wadhwani, K. C., Caspers-Velu, L. E., Murphy, V. A., Smith, Q. R., Kador, P. F., and Rapoport, S. I. (1989). Prevention of nerve edema and increased bloodnerve barrier permeability-surface area product in galactosemic rats by aldose reductase or thromboxane synthetase inhibitors. Diabetes 38, 1469-1477. doi: 10.2337/diab.38.11.1469

Wang, F., Zhou, F., Kruh, G. D., and Gallo, J. M. (2010). Influence of blood-brain barrier efflux pumps on the distribution of vincristine in brain and brain tumors. Neuro Oncol. 12, 1043-1049. doi: 10.1093/neuonc/noq056

Wang, Q., Yang, H., Miller, D. W., and Elmquist, W. F. (1995). Effect of the pglycoprotein inhibitor, cyclosporin A, on the distribution of rhodamine-123 to the brain: an in vivo microdialysis study in freely moving rats. Biochem. Biophys. Res. Commun. 211, 719-726. doi: 10.1006/bbrc.1995.1872

Conflict of Interest Statement: The authors declare that the research was conducted in the absence of any commercial or financial relationships that could be construed as a potential conflict of interest.

Received: 26 September 2013; accepted: 09 October 2013; published online: 04 November 2013.

Citation: Adkins CE, Mittapalli RK, Manda VK, Nounou MI, Mohammad AS, Terrell TB, Bohn KA, Yasemin C, Grothe TR, Lockman JA and Lockman PR (2013) P-glycoprotein mediated efflux limits substrate and drug uptake in a preclinical brain metastases of breast cancer model. Front. Pharmacol. 4:136. doi: 10.3389/fphar.2013.00136 
This article was submitted to Pharmacology of Anti-Cancer Drugs, a section of the journal Frontiers in Pharmacology.

Copyright (c) 2013 Adkins, Mittapalli, Manda, Nounou, Mohammad, Terrell, Bohn, Yasemin, Grothe, Lockman and Lockman. This is an open-access article distributed under the terms of the Creative Commons Attribution License
(CC BY). The use, distribution or reproduction in other forums is permitted, provided the original author(s) or licensor are credited and that the original publication in this journal is cited, in accordance with accepted academic practice. No use, distribution or reproduction is permitted which does not comply with these terms. 